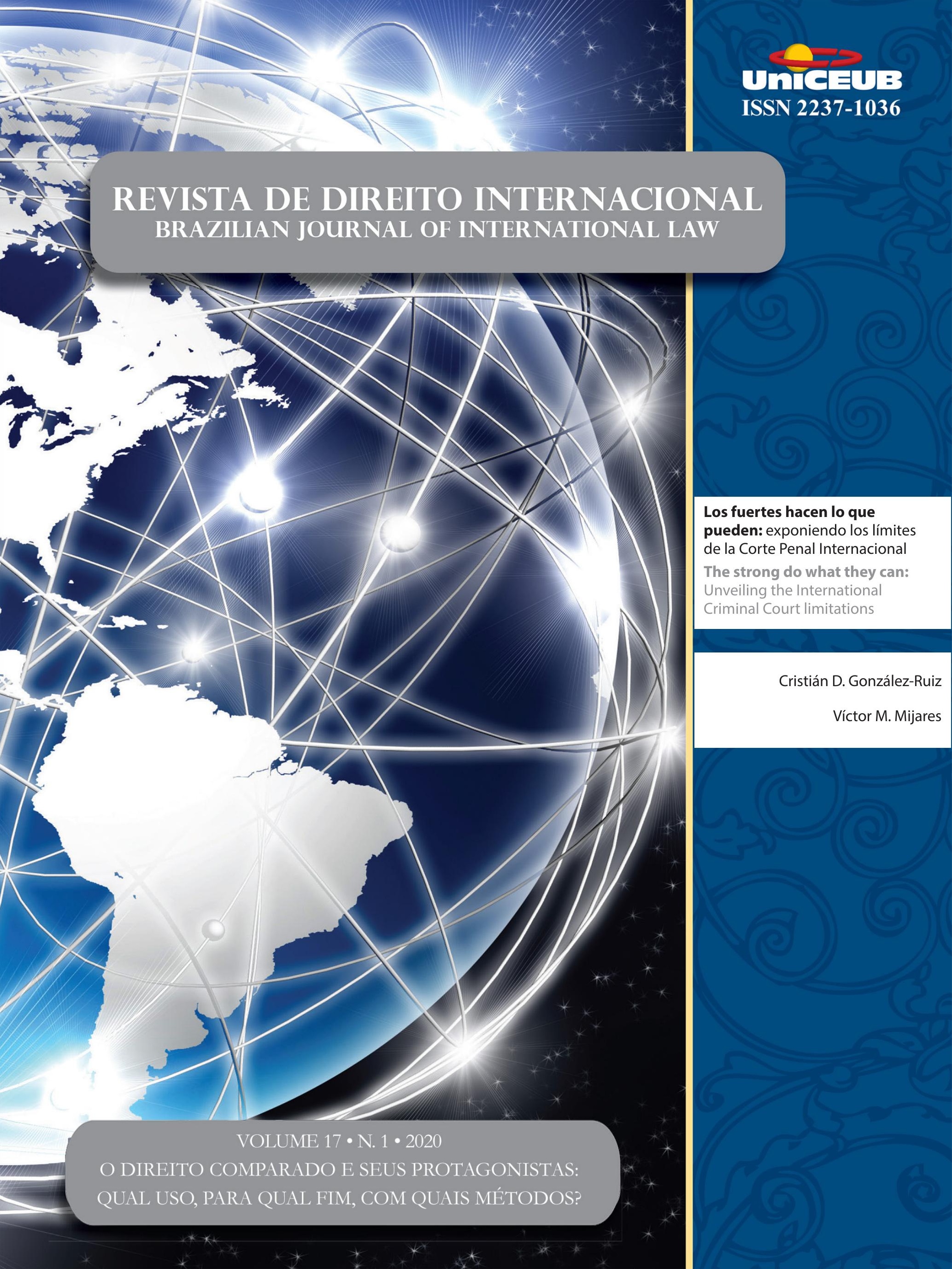


Parte I - O Direito comparado e seus Protagonistas: QuAL uso, PARA QUAL FIM, COM QUAIS MÉTODOS? .....................................................................1

EDITORIAL ............................................................................................................... 3

"Tudo o que precisamos fazer é ter certeza de que continuaremos conversando".............................. 3

Gustavo Cerqueira e Patrícia Perrone Campos Mello

Direito comparado E METOdologia $\quad$................................................... 6

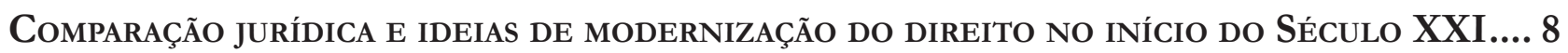
Gustavo Cerqueira

O DIREITO COMPARADO: ESFORÇO DE RESGATE HISTORIOGRÁFICO E DE PROBLEMAS METODOLÓGI$\cos$

Arnaldo Sampaio de Moraes Godoy e Gustavo Fereira Ribeiro

Direito Comparado e Política: Reflexões Necessárias .42

Raphael Carvalho de Vasconcelos e Deo Campos Dutra

Direito comparado no Brasil

L'originalité du Droit Brésilien et le Droit Comparé .57

Arnoldo Wald

LEI DA BOA RAZÃo E COMPARATISMO JURÍDICO NA DOUTRINA CIVILISTA BRASILEIRA DE 1850 A 1880

Alan Wruck Garcia Rangel

O STF EM REDE? QUANTO, COMO, COM QUE ENGAJAMENTO ARGUMENTATIVO O STF USA PRECEDENTES ESTRANGEIROS EM SUAS DECISÕES?

Patrícia Perrone Campos Mello e Felipe Meneses Graça 
Suprema Imprecisão: a metodologia em Direito Constitucional Comparado E as deficiênCias Em Seu uso pelo Supremo Tribunal Federal

Alonso Freire e Hugo Sauaia

Direitos comparados

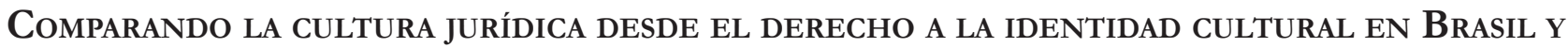

CHILE 145 Juan Jorge Faundes e Fabian Le Bonniec

O Divino e o Racional no Direito: notas para um diálogo entre sistemas jurídicos 181 Salem Hikmat Nasser e José Garcez Ghirardi

Regulação do discurso de Ódio: análise comparada em países do Sul Global 196 Jane Reis Gonçalves Pereira, Renan Medeiros de Oliveira e Carolina Saud Coutinho

Parte II - Outros temas

LA OTRA CARA DE LA MONEDA: PROTECCIÓN CONSTITUCIONAL DE LA EMPRESA, EL EMPRENDIMIENTO Y LA LIBRE COMPETENCIA EN CHILE y COLOMBiA

Juan Pablo Díaz Fuenzalida e Juan Sebastián Villamil Rodríguez

The European Court of Human Rights Decision on there 'Burqa Ban'and the CritiCal analysis of the Pragmatic experimental logic 258 Flavianne Fernanda Bitencourt Nóbrega e George Browne Rego

Direitos humanos das deslocadas ambientais e os impactos da Usina de Belo Monte: da EXPLORAÇÃO AMAZÔNICA À SUBJUGAÇÃO FEMININA

Thais Silveira Pertille e Letícia Albuquerque

Solução de Controvérsias em Acordos de Investimento: as experiênCias do CPTPP, CETA E DOS ACFIs

Fábio Morosini, Vivian Daniele Rocha Gabriel e Anastacia Costa

50 anos dos “direitos da Criança” na Convenção Americana de Direitos Humanos: a HISTÓRIA DO ARTIGO 19 311

Sven Peterke e Paloma Leite Diniz Farias 
EMPRESAS E DIREITOS HUMANOS: COMPARTILHANDO VALOR E RESPONSABILIDADES .325 Melina Girardi Fachin

CONTEMPORARY RESPONSES TO BUSINESSES' NEGATIVE HUMAN RIGHTS IMPACT 341 Andres Felipe Lopez

Human Right to LABOR PROTECTION IN UKRAINE: CURRENT SITUATION AND THE PROSPECTS OF IMPLEMENTATION OF INTERNATIONAL RULES 363 Nina Daraganova

International Regulation and Global Governance: The EU influential Method IN TIMES OF NORMATIVITY CHANGE 373 Gabriela Hühne Porto, Paula Wojcikiewicz Almeida e Juliana Maia F. A. Netto

Tecnologias digitais e o comércio de bens E SERviços na OMC/Digital. 391 Umberto Celli Junior

Los fuertes hacen lo QUe PUeden: exponiendo los límites de la Corte Penal InternaCIONAL .406

Cristián D. González-Ruiz e Víctor M. Mijares

Human Rights, Humanitarian Law and State Power 418 Renata Nagamine e João Roriz

Regional integration in the South Pacific: Challenges for Public governance .433 Joanna Siekiera

Parte III - Resenhas 443

RESENHA DA OBRA

“Democracia e policentrismo do poder", de Murilo Gaspardo 445 Angela Limongi Alvarenga Alves 


\section{Los fuertes hacen lo que pueden: exponiendo los límites de la Corte Penal Internacional*}

\section{The strong do what they can: Unveiling the International Criminal Court limitations}

Cristián D. González-Ruiz **

Víctor M. Mijares***
* Recebido em 20/10/2019

Aprovado em 04/06/2020

** Politólogo y candidato al grado de abogado de la Universidad de los Andes. Estudiante de la maestría en derecho internacional de la misma universidad y analista jurídico en la firma de derecho penal Prias Cadavid Abogados. Correo electrónico: cd.gonzalez11@uniandes. edu.co; cgonzalez@priascadavid.com

Political scientist (B.A) and candidate to LL.B degree at University of los Andes Law School. Student of the International Law Master's degree (LL.M) at the same university. Juridical analyst at the criminal law firm Prias Cadavid Abogados. Email: cd.gonzalez11@uniandes. edu.co; cgonzalez@priascadavid.com

*** Profesor Asistente de Ciencia Política en la Universidad de los Andes, Colombia. Doctor en Ciencia Política por la Universidad de Hamburgo, Alemania, y Especialista en Derecho y Política Internacionales por la Universidad Central de Venezuela. Correo electónico: vm.mijares@uniandes.edu.co

Assistant Professor of Political Science at the Universidad de los Andes, Colombia. He holds a Ph.D. in Political Science from the Universität Hamburg, Germany, and a Specialist degree in International Law and Politics from the Universidad Central de Venezuela. Email: vm.mijaresniandes.edu.co

\section{Resumen}

La Corte Penal Internacional es el mayor esfuerzo en la búsqueda de justicia universal para crímenes de lesa humanidad. No obstante su valor y el empeño en hacerla funcionar, su eficacia en la producción de sentencias muestra resultados mixtos. ¿Cuáles son las condiciones que limitan el desempeño de la Corte? Este artículo ofrece respuestas a partir de un análisis cualitativo comparado de los casos tratados por la Corte Penal Internacional, considerando factores internos y externos. El resultado es desalentador para las aspiraciones de efectividad de la justicia penal internacional, pues la evidencia señala que los acusados que cuentan con el apoyo de grandes potencias o que obtiene la victoria interna y se controlan importantes recursos naturales tienden zafarse de las consecuencias de sus crímenes. El artículo contribuye a la literatura sobre cortes internacionales proveyendo evidencia sobre las dificultades que enfrentan para encauzar a violadores de derechos humanos desde el poder.

Palabras clave: Corte Penal Internacional, análisis cualitativo comparado, derechos humanos, crímenes de guerra, realismo

\section{Abstract}

The International Criminal Court is the greatest effort in the search of universal justice for crimes against humanity. Despite its value and the effort to make it work, its effectiveness in the production of sentences shows mixed results. What are the conditions that limit the Court performance? This article offers answers based on a qualitative comparative analysis of the cases treated by the International Criminal Court, considering internal and external factors as well. The result is discouraging for the aspirations of effectiveness of international criminal justice, as the evidence indicates those offenders who have the support of great powers or who obtain internal victory and control important natural resources tend to get rid of the consequences of their crimes. The article contributes to the literature on international courts by providing evidence on the difficulties they face in prosecuting human rights violators holding power positions.

Keywords: International Crime Court, qualitative comparative analysis, human rights, war crimes, realism. 


\section{Introducción}

La conformación de la Corte Penal Internacional (CPI) rompe con presunciones propias del Derecho Internacional Público, en tanto se distancia de la tradición estato-céntrica al considerar como sujetos internacionales a las personas y no sólo los Estados, ante un tribunal cuya jurisdicción es permanente y universal. ${ }^{1} \mathrm{Y}$ en segundo lugar, rompiendo el principio de soberanía estatal absoluta, dado que, formalmente, la CPI está facultada para perseguir y castigar incluso a ciudadanos de Estados que no son parte de los que han ratificado su Estatuto Constituyente (art. 13, literal b). Así, se infiere que la creación de un tribunal como la CPI rompe con los principios básicos del realismo en las relaciones internacionales en el ejercicio del imperio de la razón sobre la fuerza, dónde los Estados -y sus líderes- nada podrán hacer ante un cuerpo colegiado, imparcial e independiente que velará por la justicia internacional y la protección del bien jurídico humanidad ante la comisión de los más atroces crímenes².

A la luz del anterior supuesto, esta investigación evalúa si el ejercicio de la CPI se trata de un comportamiento imparcial donde los factores políticos tengan baja incidencia en la producción de sentencias o si, por el contrario, el conjunto de intereses de los Estados podría tener alguna incidencia en el ejercicio del citado tribunal. La importancia teórica del escrito radica en su ubicación en la literatura de cortes y Relaciones Internacionales (RRII), igualmente relevante para el derecho y la ciencia política. Para el primero, en tanto en términos de eficacia del derecho penal internacional. Y para la segunda, en tanto contribuye al entendimiento de las relaciones de poder entre instituciones internacionales, Estados y actores sub- y trans-nacionales.

Además, el estudio de la CPI, como caso particular, reviste especial importancia, dadas las circunstancias

1 En los casos donde la jurisdicción de la Corte Penal Internacional sea activada después de la petición originada en el marco del CS. Para ver más sobre el rompimiento del paradigma del estato centrismos en el derecho internacional público ver Johns, Leslie. “The Diverging Theory and Practice of International Law." En Encyclopedia of Empirical International Relations Theory, 513-35. Oxford: Oxford University Press, 2018 y Quesada Alcalá, Carmen. La Corte Penal Internacional Y La Soberanía Estatal. Tirant Monografías, 383. Valencia: Tirant Lo Blanch, 2005.

2 Werle Gerhard, Florian Jessberger, Claudia Cárdenas Aravena, Julia Geneuss, and María Gutiérrez Rodríguez. Tratado De Derecho Penal Internacional. Tercera Edición ed. Tirant Lo Blanch. Tratados. Valencia: Tirant Lo Blanch, 2017. que rodean su funcionamiento en el sistema internacional, tales como su característica de tribunal internacional que juzga personas y no Estados, además de su vocación de permanencia. Así también, bajo la consideración de la renuencia de algunos Estados a aceptar su jurisdicción e incluso el retiro de algunos de los signatarios originales, en medio de un contexto político contemporáneo que pareciera ser renuente al funcionamiento de las instituciones internacionales. De igual forma, pretendemos aplicar los planteamientos de tres enfoques teóricos de las RRII al análisis del funcionamiento de la CPI, a saber, realismo, liberalismo y escuela inglesa, lo cual contribuirá a un entendimiento complejo del comportamiento de los actores internacionales en lo que atañe al funcionamiento del tribunal.

Desde la misma negociación de los estatutos fundacionales, la CPI ha enfrentado una serie de dificultades para su establecimiento. Para entonces, un bloque de países, liderados por EEUU, China, Rusia, y Arabia Saudita, abogaba por el establecimiento de una corte débil, cuyas sentencias fuesen no-vinculantes y de competencia subsidiaria a la jurisdicción nacional del país dónde se cometieren los crímenes. Otro grupo, liderado por Australia y Canadá, que defendía un tribunal fuerte, independiente de la influencia de los Estados, y cuya competencia podría ser incluso activada a través de la fiscalía del mismo aparato judicial. ${ }^{3}$ Estás tensiones reflejan en el producto final del Estatuto de Roma, puesto que las causales para iniciar las investigaciones en el marco del proceso penal se restringen a tres (art. 13):

1. Un Estado parte remite la investigación a la fiscalía en caso de que considere que se ha cometido alguno de los crímenes tipificados, es decir, como ejercicio de soberanía, dentro del margen de discrecional que ofrece el interés nacional;

2. El Consejo de Seguridad de las Naciones Unidas (CS), actuando conforme al Capítulo VII de la Carta de San Francisco, o;

3. El Fiscal decide abrir una investigación de acuerdo con lo dispuesto en el art. 15 del mismo estatuto.

De lo anterior, es evidente deducir que las causales 1 y 2 para activar la jurisdicción de la CPI, obedecen a una lógica de soberanía estatal e intereses de los países poderosos del sistema internacional, mientras que la úl-

\footnotetext{
Werle, Gerhard, Florian Jessberger, Claudia Cárdenas Aravena, Julia Geneuss, and María Gutiérrez Rodríguez. Tratado De Derecho Penal Internacional. Tercera Edición ed. Tirant Lo Blanch. Tratados. Valencia: Tirant Lo Blanch, 2017.
} 
tima dota a la Corte para ejercer una relativa autonomía fortalecida en su propio sistema institucional. ${ }^{4}$

Sin embargo, los problemas de la Corte no terminaron allí. Una vez la CPI inicia su labor de investigar, juzgar y sancionar los crímenes de su competencia, varios países africanos, liderados por Sudáfrica y Kenia en el seno de la Unión Africana (UA), iniciaron el cuestionamiento de la legitimidad del tribunal en términos de la supuesta injusticia que representa el hecho de que en más de un decenio de su vigencia, las investigaciones se hayan concentrado exclusivamente en territorio africano, ${ }^{5}$ específicamente en cuatro zonas: oriental, donde se encuentran los casos ocurridos en Sudán y Kenia, caracterizados por la negativa de los Estados por colaborar con la CPI; central, que comprende el territorio de las regiones de Ituri y Kivú en la República Democrática del Congo (RDC), Uganda, y República Centroafricana (RCA), que en su mayoría comprende los conflictos residuales del genocidio ruandés de 1994, el conflicto entre la Séleka y Anti-Balaka en la RCA, y el conflicto con el Lord's Liberation Army en Uganda; occidental, representada por Costa de Marfil; y norte, comprendida por Libia, caracterizada por la guerra civil que azota al país desde 2011.

En consecuencia, el creciente malestar de los países africanos también ha minado el funcionamiento de la CPI, en discursos que van desde el neocolonialismo hasta el ataque de las instituciones liberales de derecho, que incluso, han sido adoptados por las defensas técnicas de los acusados, ${ }^{6}$ que han optado por argumentos de ruptura para desacreditar los supuestos y valores que defiende la CPI. De igual forma, varios países han propuesto que el Tribunal Africano de Derechos Humanos prive de competencia a la Corte, o a la creación incluso de un tribunal penal permanente, conformado abogados africanos se encargue de las funciones de la CPI.

Este tipo de dificultades, que a simple vista no goza

\footnotetext{
Cassese, Antonio, Paola Gaeta, and John R. W. D Jones. The Rome Statute of the International Criminal Court: A Commentary. Oxford, England: Oxford University Press, 2002.

5 Para profundizar en este debate Arnould, Valérie. A Court in Crisis?: The ICC IN Africa, and Beyond. Report. Egmont Institute, 2017. 15-18. http://www.jstor.org/stable/resrep06704.8.

; Keppler, Elise. "Managing Setbacks for the International Criminal Court in Africa." Journal of African Law 56, no. 1 (2012): 1-14. http://www.jstor.org/stable/41709948. 2012; Frahm, Ole. Report. Istituto Affari Internazionali (IAI), 2015. http://www.jstor.org/stable/resrep09759.
}

de relación mutua, en realidad se conecta a través de un mismo cuestionamiento: ¿se trata el ejercicio de la CPI de un mero juicio espectáculo y/o simbólico a interés de los países poderosos, o estamos ante una institución objetiva, que respeta los fundamentos de la legalidad y únicamente busca la protección del bien jurídico de la humanidad? O en términos de la literatura de justicia transicional, ¿la CPI, como tribunal internacional permanente, en su actuación obedece a la lógica de la justicia política ${ }^{7}$ o justicia legal?

Sin embargo, para responder a dichos interrogantes, es necesario primero preguntarse ¿bajo qué condiciones produce sentencias la CPI? Esta pregunta obedece a una lógica de efectividad entendida a partir de la producción de decisiones judiciales por parte de la Corte, como parte de un doble mensaje, punitivo y disuasorio de conductas individuales que constituyan la comisión de los delitos tipificados por el Estatuto de Roma.

No obstante, es necesario reconocer que reciente literatura de cortes y RRII ha criticado éste enfoque argumentando que la producción de sentencias no obedece a otra lógica que a la de una variable espuria o, en el mejor de los casos, proxy. ${ }^{9}$ Sin embargo, consideramos que esta es la mejor manera de problematizar la coyuntura internacional ante la dificultad de operacionalización de otros tipos de enfoques metodológicos, como la de es-

Esto se evidencia en la preparación ex ante a posibles argumentaciones de ruptura en la defensa de los imputados de dichos crímenes (defensa con argumentos del tipo "tú hiciste las mismas cosas que yo, en incluso peor, ¿qué autoridad tienes para juzgar mis actos?), la restricción de elementos probatorios que cuestionen la formación de tribunales de este estilo, la utilización de la justicia penal extraordinaria como espectáculo para intentar legitimar las decisiones tomadas a través del derecho, una seguridad jurídica que depende del soberano, la invención de chivos expiatorios, y finalmente la impunidad de los crímenes cometidos por el bando vencedor de la guerra. Douglas, Lawrence. The Shrunken Head of Buchenwald: Icons of Atrocity at Nuremberg. Representations, (63), 39-64. doi: $10.2307 / 2902917.2018$

8 Destaca la importancia de principios propios del Estado de Derecho como una judicatura independiente de la administración, jueces imparciales, el respeto al principio de legalidad, es decir, a ser juzgado con base en crímenes preexistentes, el respeto al debido proceso y al rule of law, la presunción de inocencia, la presencia de elementos que permitan la controversia probatoria, segunda instancia, y un modelo adversarial en el litigio que permita contradicción entre las partes, en síntesis, se trata de un concepto de justicia legal. Bass, Gary. Stay the Hand of Vengeance: The Politics of War Crimes Tribunals. Princeton: Princeton University Press. 2014

9 Shany, Yuval. Assessing the Effectiveness of International Courts: A Goal-Based Approach. American Journal of International Law 106, Nr. 2: 225-270. doi:10.5305/amerjintelaw.106.2.0225, 2012. 
tablecer una evaluación entre la coincidencia de los intereses de las Cortes versus la de los Estados miembros, o la satisfacción debida de las metas de dichas instituciones, que además incluiría un enfoque normativo.

\section{Análisis Cualitativo Comparado}

Para analizar las condiciones bajo las cuales la CPI sentencia se ve imposibilitada de producir sentencia, el diseño metodológico escogido para llevar a cabo esta investigación es el comparado, tomando como método el análisis cualitativo comparado (Qualitative Comparative Analysis o QCA), a través del cual se busca conocer cuáles son las condiciones suficientes y necesarias para la producción de un resultado específico, en concreto, la producción (o ausencia) de sentencia por parte de la CPI. El QCA intenta descubrir el mecanismo de causalidad involucrado entre una serie de variables $(\mathrm{X})$ y la producción de un resultado $(\mathrm{Y})$ en términos de condiciones suficientes y necesarias. Entonces, a partir de la selección de un número de casos, muestra representativa del objeto de estudio, se construye el método a través de cuatro fases, a saber: a) la construcción de una tabla comparativa para organizar la información cualitativa e identificar las posibles condiciones necesarias; b) articular la teoría tipológica y clasificar los casos en función de dicha metodología a través de tablas de verdad; c) un análisis de los casos contrafactuales, es decir, de aquellos sobre los que no hay ejemplos en la realidad, y; d) la reducción del número de configuraciones suficientes a través de un proceso de minimización lógica ${ }^{10}$.

Lo anterior condice al análisis de las condiciones suficientes y necesarias. Las condiciones necesarias se identifican a partir de la selección de los casos positivos, es decir aquellos donde se produjo como resultado la variable dependiente $(\mathrm{Y})$ y las condiciones causales $(\mathrm{X})$ comunes a todos ellos. Por otra parte, para mirar la suficiencia, el proceso se torna más complejo y será obligatorio que el investigador construya un índice de consistencia que contenga la proporción de casos en cada grupo que ofrece un resultado positivo. Así, en el caso de la investigación en curso, lo que se pretende es descubrir el nexo de causalidad que vincularía una serie de variables políticas $(\mathrm{X} 1, \mathrm{X} 2, \mathrm{X} 3 \ldots)$ a una consecuencia

10 Pérez-Liñán, A. El método comparativo y el análisis de configuraciones causales. Latin American Review of Comparative Politics, 3,. 2010. jurídica, que es la orden de una sentencia por parte de la CPI $(\mathrm{Y})$.

En consecuencia, el objeto de estudio serán los casos sobre los cuales la CPI ha asumido competencia en tanto a la comisión de crímenes de lesa humanidad, genocidio y crímenes de guerra para el juzgamiento y condena de los presuntos responsables. Por otra parte, los casos serán elegidos con base en el método de diferencia directo, mediante el cual se seleccionan casos positivos y negativos donde todos los casos que presentan el resultado de interés (sentencia por parte de la CPI) deben compartir una característica explicativa en común, mientras que los negativos carecen de dicho atributo, ello con el fin de evitar posible sesgos en la selección de los casos e identificar las variables necesarias pero no suficientes para Y.

En la aplicación del QCA la variable dependiente fue dicotómica y definida como la producción de sentencias por parte de la CPI. Esta variable fue medida a través de la información que se obtenga de la página web oficial de la institución donde aparece el actual estatus de cada uno de los procesos. ${ }^{11}$ Entonces se determinó $\mathrm{Y}=1$ cuando el caso haya sido terminado por una sentencia; $y, Y=0$ si el proceso sigue en curso o ha terminado de forma distinta a una sentencia.

\section{V1: Presencia de recursos en el país del acusado.}

Como afirma Rudolph ${ }^{12}$, las instituciones internacionales son una manifestación del poder, y no solamente un producto del ejercicio del mismo. Es así como, en el marco de una actuación judicial por parte de un organismo internacional como la CPI, los intereses de los países miembros y no-miembros del estatuto de Roma se ven manifiestos tanto en el diseño institucional como en la práctica de las actividades de esta, en el caso concreto, juzgar y sancionar los delitos tipificados en el estatuto constitutivo.

Asimismo, la literatura de instituciones internacionales ha demostrado que cuando un país posee amplios recursos naturales, es capaz de asumir un rol de liderazgo en el desarrollo de las actividades de la institución, o al menos influir en la toma de decisiones conjunta que

\footnotetext{
11 https://www.icc-cpi.int/

12 Rudolph, Christopher. Power and Principle: The Politics of International Criminal Courts. Ithaca; London: Cornell University Press, 2017. http://www.jstor.org.ezproxy.uniandes.edu.co:8080/ stable/10.7591/j.ctt1qv5q0z.
} 
en el marco de ella sea hecha ${ }^{13}$. Este Estado utilizará los vínculos económicos que tenga con el resto sus copares, en algunos casos más poderosos, para ejercer influencia en la toma de decisiones de la respectiva institución, a veces llevándolas al objetivo de la satisfacción del interés nacional del actor influyente sobre el objetivo conjunto de la institución.

Así, a mayor cantidad de recursos en el país del que provenga el acusado, mayor influencia en las instituciones internacionales para que el proceso no culmine en algún tipo de sentencia. Esta variable será medida a través de índices econométricos sobre la producción de los siguientes recursos en el país de origen: gas natural, petróleo, oro, uranio y coltán. Así como la cantidad de agua dulce presente en el país. Esto provisto por índices del Banco Mundial, el Fondo Monetario Internacional y bases de datos privadas como el World Resources Institute. Se consideró que X1=1 cuando el país del cual provenga el acusado se encuentre dentro de los 10 Estados con mayores reservas a nivel mundial de cualquiera de los recursos mencionados en el párrafo anterior y X1 $=0$ de lo contrario.

V2: Apoyo exterior por parte de algún miembro del CS al bando al que pertenece el acusado

Hay al respecto dos grandes corrientes teóricas que intentan describir el fenómeno del apoyo de miembros del CS a los vinculados a un proceso penal por parte de la CPI. En primer lugar, aquellos que defienden definirlo en cuanto al interés en términos de poder ${ }^{14} \mathrm{y}$ aquellos que defienden la teoría de la dependencia.

En cuanto a los primeros, entienden que los Estados actúan salvaguardando su interés nacional y ejercerán influencia para favorecer ya sea el statu quo o el cambio en la distribución del poder en el sistema global de

\footnotetext{
13 Rios de tinta han sido escritos al respecto del poder que países ricos ejercen en la formación, funcionamiento, éxito o fracaso de una organización internacional. Ver por ejemplo, Olatunde J. B. Ojo. "Nigeria and the Formation of ECOWAS." International Organization 34, no. 4 (1980): 571-604. http://www.jstor.org.ezproxy.uniandes.edu.co:8080/stable/2706513; Nelson, Marcel. "Institutional Conflict and the Bolivarian Revolution: Venezuela's Negotiation of the Free Trade Area of the Americas." Latin American Perspectives 40, no. 3 (2013): 169-83. http://www.jstor.org.ezproxy.uniandes.edu.co:8080/stable/23466010; ELLNER, STEVE. Journal of Latin American Studies 44, no. 1 (2012): 202-04. http://www.jstor.org. ezproxy.uniandes.edu.co:8080/stable/41349744, entre otros.

14 Rudolph, Christopher. Power and Principle: The Politics of International Criminal Courts. Ithaca; London: Cornell University Press, 2017. http://www.jstor.org.ezproxy.uniandes.edu.co:8080/ stable/10.7591/j.ctt1qv5q0z.
}

acuerdo con su propia conveniencia, es decir, los réditos que ello le represente. Dicho interés se manifiesta tanto en el diseño institucional de organismos como la CPI, como en una inminente influencia para las posteriores decisiones tomadas por la misma.

En consecuencia, al ser la CPI una institución que claramente desafía los presupuestos de la soberanía westfaliana ${ }^{15}$, el balance de poder existente en el marco del CS al no estar supeditada la Corte exclusivamente a las órdenes de éste, y la posibilidad incluso de investigar juzgar y sancionar incluso ciudadanos de los países que no hacen parte del tratado (entre ellos tres miembros del CS: Rusia, China y EEUU). Entonces, será el interés de dichos Estados minar el funcionamiento de la CPI, apoyando al indiciado o acusado tanto en las dinámicas de la guerra, de acuerdo con el interés de la gran potencia en el territorio donde se lleven a cabo las operaciones, tanto como en las respectivas instancias internacionales dónde los líderes de las facciones armadas son llamados a rendir cuentas ante la justicia penal internacional.

Por otra parte, de acuerdo con Goodlife et al. ${ }^{16}$, los gobiernos dependen de otros en una red en la que pertenecen otros países y gobiernos. En consecuencia, la decisión que tome un Estado afectará la toma de decisiones de sus copares y otros actores en el marco del conflicto armado en el cual se desarrollan los casos de conocimiento por parte de la CPI, especialmente si se trata de un Estado poderoso.

A mayor influencia que tengan los miembros de la red en el ejercicio de la presión, es más probable que el Estado cambie sus actuaciones. Entonces, si un país que hace parte del CS es miembro de la misma red a la cual pertenece el Estado del cuál proviene el acusado, manifiesta su hostilidad o desinterés a las decisiones y medidas adoptadas por la CPI, lo normal es que el segundo Estado adopte medidas que sigan el patrón del actor más poderoso. Esta hostilidad sería causada por la existencia de objetivos geoestratégicos, económicos o políticos en el país del cuál proviene el acusado por parte de un miembro del CS.

\footnotetext{
15 Krasner, Stephen D. Soberanía, hipocresía organizada. Paidós: Barcelona, 2001

16 Goodliffe, Jay, Darren Hawkins, Christine Horne, and Daniel L. Nielson. "Dependence Networks and the International Criminal Court.” International Studies Quarterly 56, no. 1 (2012): 131-47. http:// www.jstor.org.ezproxy.uniandes.edu.co:8080/stable/41409827.
} 
Entonces, combinando las dos corrientes teóricas, esta variable se va a medir conforme a los entendimientos que dicha facción haya suscrito con Rusia, China, Reino Unido, EEUU o Francia. Esta información es provista por las páginas web de los respectivos ministerios de Relaciones Exteriores o su equivalente de los países miembros permanentes del CS, páginas web de las empresas en cuestión, así como datos de organizaciones entre las que se encuentran la UA y la ONU. Así, $\mathrm{X} 2=1$ cuando el país del acusado haya suscrito al menos un (1) acuerdo (sea tratado de derecho internacional público, o alianza geopolítica en caso de tratarse de una facción no estatal) con países del CS, de lo contrario, $\mathrm{X} 2=0$.

\section{V3: Pertenencia del acusado al bando que ganó la guerra}

Siguiendo a Bass ${ }^{17}$ y a Rudolph ${ }^{18}$, cuando se trata de tribunales penales internacionales, es necesario identificar dos nociones fundamentales: la de justicia politica y la de justicia legal. La primera hace referencia a los así denominados "juicios espectáculo" donde quién administra la justicia es un tribunal políticamente sobre determinado y cuyo resultado no es contingente. En otras palabras, de trata de un ejercicio de justicia vengativa y directa de los vencedores sobre los vencidos, falsamente legitimada a través de un tribunal que está encomendado de ejercer fallos en derecho.

Por otra parte, la justicia legal hace referencia a aquellos procesos en los cuales unos jueces imparciales siguen atentamente los derechos consagrados en el instrumento legal previamente establecido que regirá el proceso, especialmente, las reglas del debido proceso y el derecho fundamental que este otorga a los perseguidos penales, de la contradicción y defensa frente a sus acusadores. Cuando el acusado haya pertenecido al bando ganador de la guerra en cuestión, el Estado dificultará la consecución de pruebas por parte de la fiscalía de la CPI, le restará contingencia al resultado y causará que ante la inexistencia de un acervo probatorio la presunción de inocencia prevalezca. De igual forma, el estatuto procesal de la CPI otorga al respeto de una garantía al

\footnotetext{
17 Bass, Gary. Stay the Hand of Vengeance: The Politics of War Crimes Tribunals. Princeton: Princeton University Press. 2014; Bass, Gary J. “Atrocity \& Legalism.” Daedalus 132, no. 1 (2003): 73-82. http:/ / www.jstor.org/stable/20027824.

18 Rudolph, Christopher. Power and Principle: The Politics of International Criminal Courts. Ithaca; London: Cornell University Press, 2017. http://www.jstor.org.ezproxy.uniandes.edu.co:8080/ stable/10.7591/j.ctt1qv5q0z.
}

debido proceso que ha sido en muchas ocasiones una traba para llevar adelante los propios procesos, y es que la persona que no haya sido capturada o no haya comparecido voluntariamente a la Corte, no podrá ser juzgada por la misma.

Entonces, la persona o grupo que resulte victoriosa en el marco de un enfrentamiento armado, no comparecerá voluntariamente a un tribunal a enfrentarse en juicio frente a una fiscalía que ya tiene acervo probatorio suficiente al menos para inculparlo sobre la comisión de los crímenes más atroces en contra de la humanidad misma. Asimismo, si dicha facción logra hacerse con el control del Estado, la situación se tornará incluso más difícil dadas las protecciones y garantías internacionales a los jefes de Estado y altos cargos de gobierno, así como la carga política que conllevaría el hecho de capturar a alguna persona de rango alto dentro de la organización burocrática de un Estado so pretexto de llevarlo a juicio por la presunta comisión de crímenes de guerra o crímenes contra la humanidad. Esta variable será medida a partir de la identificación de los objetivos del grupo al cual pertenece el acusado como actor político, con base en fuentes primarias (documentos propios del grupo) y la consecución de los mismos en el marco de la confrontación bélica con el resto de grupos armados.

En consecuencia, cuando el actor político haya conseguido sus objetivos a través de la confrontación bélica (ej. Lograr el control del Estado, lograr un armisticio en condiciones favorables, la secesión del territorio de un país), $\mathrm{X} 3=1$. Si de lo contrario, la confrontación bélica no ha terminado o el actor político ha sido derrotado en el marco de las actividades militares, X3 $=0$.

V4: Presencia de un elemento coercitivo para los Estados de acatar las decisiones de la Corte

De acuerdo con de Nevers ${ }^{19}$, y Ratner ${ }^{20}$, si bien, a partir de 1945 las potencias mundiales y regionales, organizaciones internacionales y otros actores transnacionales han logrado desafiar la tradición de carácter consensual del Derecho Internacional Público, ejerciendo su influencia para el cumplimiento de las normas estipuladas, hoy en día, ante la falta de elementos coercitivos las decisiones que no son aceptadas por las partes en

\footnotetext{
19 De Nevers, Renee. "Imposing International Norms: Great Powers and Norm Enforcement." International Studies Review 9, no. 1 (2007): 53-80. http://www.jstor.org/stable/4621777.

20 Ratner, Steven R. "International Law: The Trials of Global Norms.” Foreign Policy, no. 110 (1998): 65-80. doi:10.2307/1149277.
} 
un litigio ante un tribunal internacional terminan sin un efecto práctico.

Por elementos coercitivos en el sistema internacional moderno, se entienden mecanismos como la imposición de sanciones ${ }^{21}$ y condenas por parte de organismos como las Naciones Unidas (tanto CS como Asamblea General), e instituciones regionales como la UE, que relegan al Estado infractor de normas, de la comunidad internacional y le imponen cargas gravosas de índole tanto económico como político al Estado infractor. Esta variable es de difícil operacionalización ante la imposibilidad de medir el grado de poder que es capaz de ejercer un Estado o una institución internacional en términos de law enforcement en el sistema internacional. Sin embargo, esta variable se medirá a partir de las sanciones impuestas al país del cuál proviene el acusado por parte del CS, el Departamento de Estado de los EEUU o la UE, a partir del inicio de la fase investigativa de un caso ante la CPI en razón del desacato a las instrucciones del tribunal.

Entonces, X5=1 si a partir de la apertura del caso las instituciones en cuestión han sancionado al menos una (1) vez al Estado del que proviene el acusado en función del desacato a las órdenes impuestas por el tribunal. De lo contrario, $\mathrm{X} 5=0$.

Teniendo en cuenta la operacionalización de las variables dependiente e independientes, fue construida una tabla de verdad que fue analizada con la hoja de cálculo de Lasse Cronqvist, ${ }^{22}$ diseñada para ejecutar la identificación de las variables independientes que sean necesarias y suficientes para la producción del resultado relevante, es decir, cuando $\mathrm{Y}=0$, o la CPI no emite sentencia, con un $n$ equivalente a 43 producto de los casos en los cuales la CPI ha intervenido, so pretexto de la presunta comisión de los crímenes tipificados en el estatuto de Roma.
Tabla 1. Tabla de Verdad y Análisis Cualitativo Comparado

\begin{tabular}{|c|c|c|c|c|c|}
\hline Casos & recursos & apoyo & victoria & coerción & sentencia \\
\hline $\begin{array}{l}\text { Sang, Ruto, } \\
\text { Matthauora, } \\
\text { Kosgey, Kenyatta, } \\
\text { Gicheru, Borasa, } \\
\text { Ali, Mohammed }\end{array}$ & 0 & 0 & 1 & 0 & 0 \\
\hline $\begin{array}{l}\text { Otti, Ongwen, } \\
\text { Okot, Lukwiya, } \\
\text { Kony }\end{array}$ & 0 & 1 & 0 & 0 & C \\
\hline Abu Garda & 1 & 0 & 0 & 0 & 0 \\
\hline $\begin{array}{l}\text { al Hassan, } \\
\text { al Mahdi, Al } \\
\text { Werfalli, Banda, } \\
\text { Ntaganda, } \\
\text { Mbarushimana, } \\
\text { Magenda } \\
\text { Kabongo, } \\
\text { Lubanga, Kilolo, } \\
\text { Katanga, Jerbo, } \\
\text { Gbagbo, Simone, } \\
\text { Gbagbo, Laurent, } \\
\text { Gaddafi, Said al } \\
\text { Islam, Gaddafi } \\
\text { Muammar, } \\
\text { Ble Goude, Al } \\
\text { Rahman, Ali Abd } \\
\text {, Al-Werfalli, } \\
\text { Al-Tuhamy, Al } \\
\text { Senussy, Ag } \\
\text { Abdoul Aziz al } \\
\text { Mohammed }\end{array}$ & 1 & 1 & 0 & 0 & C \\
\hline $\begin{array}{l}\text { Yekatom, } \\
\text { Mudacumura }\end{array}$ & 1 & 1 & 0 & 1 & C \\
\hline $\begin{array}{l}\text { Ngudjdo Chui, } \\
\text { Hessein, Abdel, } \\
\text { Harun, Bemba } \\
\text { Gambo, Arido }\end{array}$ & 1 & 1 & 1 & 0 & C \\
\hline
\end{tabular}

\section{\# Implicantes:}

2

recursos * Sang, Ruto, Matthauora, Kosgey, Kenyatta, VICTORIA Gicheru, Borasa, Ali, Mohammed

Sang, Ruto, Matthauora, Kosgey, Kenyatta, Gicheru, Borasa, Ali, Mohammed, Abu

\# Soluciones: 1 Garda

APOYO

\section{Interpretación de resultados}

El QCA arroja como condición necesaria a la producción del resultado relevante el apoyo de los países miembros del CS al bando al cual pertenece el acusado. De igual forma, son implicantes, o condiciones suficientes conjuntas, la presencia de recursos en el país del acusado y la victoria del bando al cual pertenece el acusado

El desarrollo del derecho penal internacional, tal como lo conocemos actualmente, es una creación re-

\footnotetext{
21 Para profundizar en el tema de las sanciones como mecanismo de castigo internacional, ver Nossal, Kim Richard. "International Sanctions as International Punishment." International Organization 43, no. 2 (1989): 301-22. http://www.jstor.org.ezproxy.uniandes.edu. co:8080/stable/2706704.

22 Cronqvist, Lasse. QCA Add-In. Version 1.1. Trier, 22.02.2019. https://www.qca-addin.net
} 
ciente. Si bien los aliados congregados en la Sociedad de Naciones propusieron la constitución de un tribunal capaz de juzgar los crímenes presuntamente cometidos por el Káiser Guillermo, fue solamente hasta la Segunda Guerra Mundial que se celebraron juicios por crímenes de lesa humanidad, Núremberg ${ }^{23}$. Una vez finalizado el juicio contra los nazis, y ante la ausencia de un enemigo común, la URSS y los EEUU se enfrentaron en la Guerra Fría, lo que dejó a un lado la creación de un aparato judicial internacional que se encargara de castigar la comisión de ciertos delitos contra la humanidad, ante la imposibilidad de llegar a un consenso sobre los delitos punibles y la forma en la que dicho tribunal actuaría.

En la inmediata post-Guerra Fría, y a raíz de las masacres de Ruanda y la intervención de la OTAN en Yugoslavia, desde Occidente se consideró necesario crear tribunales internacionales capaces de investigar y juzgar los crímenes cometidos en las dinámicas de la guerra. Ello ocurrió en un ambiente donde proliferaba la victoria de los valores liberales, la tercera ola de democratización ${ }^{24}$, e incluso la aparición de conceptos como la Responsabilidad de Proteger ${ }^{25}$. De allí derivó la idea de crear un tribunal internacional permanente ${ }^{26}$, que finalmente tras serias discusiones dogmáticas y políticas entró en funcionamiento en el año 2003, después de la promulgación del estatuto de Roma el año anterior.

Este breve recuento histórico es útil para identificar que los tribunales penales que han sido creados por la sociedad internacional, hayan sido desarrollados a partir de un contexto en el cual los valores del liberalismo parecieren imponerse sobre el realismo. Es así cómo, en el diseño institucional de tribunales como el de Núremberg, Yugoslavia o Ruanda, se constituyen principios que desafían el orden del Derecho Internacional Públi-

\footnotetext{
23 Werle Gerhard, Florian Jessberger, Claudia Cárdenas Aravena, Julia Geneuss, and María Gutiérrez Rodríguez. Tratado De Derecho Penal Internacional. Tercera Edición ed. Tirant Lo Blanch. Tratados. Valencia: Tirant Lo Blanch, 2017.

24 Schneider, Ben Ross. "Democratic Consolidations: Some Broad Comparisons and Sweeping Arguments." Latin American Research Review 30, no. 2 (1995): 215-34. http:/ / www.jstor.org.ezproxy.uniandes. edu.co:8080/stable/2503840.

25 Sampford, Charles. "A Feuerbachian Inversion: From Sovereign Rights and Subjects Duties to Citizen Rights and State Duties." Security Challenges 7, no. 4 (2011): 51-60. https://www-jstor-org.ezproxy. uniandes.edu.co:8443/stable/26467116.

26 Rudolph, Christopher. Power and Principle: The Politics of International Criminal Courts. Ithaca; London: Cornell University Press, 2017. http://www.jstor.org.ezproxy.uniandes.edu.co:8080/ stable/10.7591/j.ctt1qv5q0z.
}

co vigente, basado en la consensualidad, la personalidad jurídica exclusiva del Estado, y el principio westfaliano del cujus regio, ejus regido, base del concepto actual de soberanía para mutar en conceptos como la existencia de bienes jurídicos universalmente protegidos, o la facultad que tiene la humanidad conjunta de castigar individuos sin importar su nacionalidad. La creación de tribunales penales internacionales se trata de un genuino intento de ejercer la justicia legal para castigar a los criminales cuyos crímenes hayan afectado a la humanidad, otorgando siempre las garantías propias de un Estado de derecho como el derecho al debido proceso, la debida controversia de las pruebas aportadas, el derecho a la doble instancia, entre otras.

La CPI no ha sido la excepción. El proceso de negociación de la misma, aún bajo la protesta de grandes potencias como los EEUU, terminó formando un tribunal a priori independiente incluso del CS, facultado para que la fiscalía tome la decisión, incluso por su propia iniciativa de investigar los crímenes presuntamente cometidos, y en algunos casos capaz de juzgar incluso ciudadanos de países que no hagan parte de su estatuto constitutivo. Por otra parte, la Corte también fue facultada para librar órdenes de captura, incluso contra jefes de Estado en ejercicio de sus funciones o altos miembros del gobierno nacional protegidos bajo el régimen internacional de inmunidades, como es en el caso de Omar al-Bashir y Abu Garda, presidente y ministro de gobierno Sudanés o Blé Goudé entonces presidente de Costa de Marfil.

Sin embargo, el ejercicio de la práctica de la CPI, muestra que se trata efectivamente de un ejercicio de justicia politica. Más allá de la ya polémica discusión sobre los casos que escoge la Corte para iniciar juicios, localizados en su totalidad en el continente africano, hecho que ha sido enérgicamente protestado por los miembros de la UA, y que podría ser objeto de una nueva investigación, la aplicación del método del análisis cualitativo comparado arroja que, para que la CPI no produzca una sentencia, es apenas necesario que el acusado tenga el apoyo de alguna potencia internacional.

Ello se puede explicar por dos razones. La primera es que cuando el acusado cuenta con el apoyo de un país extranjero, dicha protección tiene un doble efecto: por una parte, al interior, le dará legitimidad a la razón por la cual su facción decidió irse a las armas, como lo dicta el ejemplo paradigmático del apoyo a los freedom fighters ${ }^{27}$

\footnotetext{
Lagon, Mark P. “The International System and the Reagan Doc-
} 
apoyados por EEUU, además de una considerable ventaja militar sobre el resto de actores políticos involucrados en la contienda en términos de armamentos, asesoría militar externa y recursos; y por otra, más relevante en la investigación, el efecto exterior es la protección que las potencias mundiales pueden otorgar a quien resulte investigado por la comisión de los delitos que estudia la CPI, dado que, por una parte, no emprenderá la fiscalía de la Corte ninguna investigación contra dicho personaje por la petición del CS bajo las facultades que el estatuto de Roma le otorga.

Pero la más importante es que la práctica judicial de la Corte demuestra que los Estados cuyo interés dista de la existencia de una CPI independiente, ejercen su influencia para que una sentencia que reafirme la tarea de la Corte en la búsqueda de la consecución de su objetivo no sea logrado. Entonces, así la CPI se trace como objetivo fundacional ejercer una labor de justicia legal, en términos de independencia judicial, lo cierto que es que este criterio todavía es extraño al ejercicio del derecho penal internacional ${ }^{28}$, donde los Estados poderosos aún ejercen influencia para restarle efectividad a la labor de la Corte. De igual forma, la suficiencia de la conjunción entre la presencia de recursos en el país del acusado y la victoria del acusado en el marco del conflicto librado, muestra de igual forma que el interés y el ejercicio de poder siguen siendo factores claves para evitar que la CPI produzca sentencia para finalizar los casos investigados.

Así es posible ver que, por ejemplo, en la RDC, RCA, Ruanda y Burundi, una sub-región rica en recursos como coltán, oro, uranio, entre otros, únicamente hayan sido condenados mediante sentencia que finaliza el conflicto, los condenados por los casos de Lubanga y Katanga, tristemente célebres por el reclutamiento forzado de niños en el marco de la Segunda Guerra del Congo y por el conflicto armado en Ituri, pero también porque sus grupos de rebeldes fueron debilitados, y la captura de ambos se produjo por una redada del gobierno de Kinshasa liderado por el presidente Kabila. Mientras que, casos como el de Omar al-Bashir en Sudán, por más que la investigación se encuentre avanzada

trine: Can Realism Explain Aid to 'Freedom Fighters?'” British Journal of Political Science 22, no. 1 (1992): 39-70. http://www.jstor.org. ezproxy.uniandes.edu.co:8080/stable/1938

28 Meron, Theodor. "Judicial Independence and Impartiality in International Criminal Tribunals." The American Journal of International Law 99, no. 2 (2005): 359-69. doi:10.2307/1562502. en los términos de una posible comisión de crímenes de lesa humanidad entre los que se encuentran homicidios, exterminación desplazamiento forzado, etc., así como crímenes de guerra como ataques deliberados e intencionales contra la población civil y genocidio, todo ello en el marco del conflicto sudanés en la región occidental de Darfur, no ha habido sentencia. Ello se podría explicar en tanto hasta 2019 el presidente de Sudan controlaba las abundantes reservas petroleras de dicho Estado, que han sido históricamente explotadas por China, además del control de las aguas sobre el caudal del Nilo, que le da soporte vital a una de las economías y potencias militares más grandes de África como lo es Egipto, todo ello sin contar las ventajas que le ofrece ser líder de un Estado, lo que incluso le permitió viajar a países miembros del estatuto de Roma, a pesar de pesar en su contra una orden de captura vigente por parte de la CPI.

\section{Conclusiones}

La CPI es una institución que sin duda ha marcado la historia internacional reciente. La simple idea de concebir un tribunal internacional permanente fuera de la institución del CS, y por lo tanto aparentemente lejos de la influencia que aquél pudiera ejercer. De igual forma, a través de su fiscalía, es capaz de iniciar incluso investigaciones de oficio contra presuntos criminales, así como igualar a las personas y los Estados como sujetos de derecho internacional. En consecuencia, a priori la CPI rompería con los principios del antiguo orden westfaliano para imponer en el mundo un nuevo sistema, de corte más kantiano, en el cual el paradigma de la soberanía y el interés nacional quedarían relegados a ser conceptos secundarios para dar paso a los ideales de justicia global y protección a cada una de las personas que habitan el planeta.

Sin embargo, desafortunadamente para quienes defienden la idea de la creación de un sistema judicial internacional fuerte, independiente y capaz de impartir justicia a los más terribles criminales, la práctica internacional muestra que hay una discrepancia entre ideas e intereses, en un claro desbalance en favor de éste último, lo que demuestra que el orden westfaliano, de soberanías estables y fuertes, así como uno donde los actores interactúan en el sistema a partir de su interés nacional. 
En consecuencia, al identificarse que los factores suficientes y necesarios para que la CPI produzca el resultado sentencia, se demuestra cómo el ejercicio de la CPI se trata de uno de justicia política, matizado por el diseño institucional más característico de la justicia legal sin dejar de ser de índole sin duda realista.

En síntesis, en palabras de Bull ${ }^{29}$, en el sistema internacional existe un orden ya establecido y que difícilmente podrá cambiar. Este está basado en las relaciones de poder que ejercen entre ellos Estados soberanos que, en una sociedad anárquica, juegan un rol importante en el balance de poder, el Derecho Internacional Público, la diplomacia, la guerra, y el diálogo entre grandes poderes. El caso de la CPI es elocuente, puesto que las naciones que conforman la sociedad internacional establecen una nueva forma de derecho internacional público con reglas que determinan la conducta de sus miembros, pero, los conceptos de balance de poder, diplomacia, guerra y diálogo entre las naciones también se hacen manifiestos al momento de ejecutar y hacer cumplir las normas del derecho internacional.

Así, aunque es éticamente deseable que dichos problemas sean solucionados a partir de un sistema judicial independiente y respetuoso de las garantías de los procesados, nuestro análisis comparado sobre la producción de sentencias penales internacionales demuestra que ello no es suficiente. Los resultados planteados ponen de relieve las limitaciones que siguen padeciendo las instituciones liberales internacionales para alcanzar una justicia objetiva, operativa y universal ${ }^{30}$ cuando los perpetradores de violaciones a los derechos humanos logran mantenerse en posiciones de poder.

\section{Referencias}

Arnould, Valérie. A Court in Crisis?: The ICC IN Africa, and Beyond. Report. Egmont Institute, 2017. 15-18. http://www.jstor.org/stable/resrep06704.8. 2017.

Bass, Gary. Stay the Hand of Vengeance: The Politics of War Crimes Tribunals. Princeton: Princeton University Press. 2014

29 Bull, H. La sociedad anárquica: un estudio sobre el orden en la política mundial. Madrid. Catarata. 1977.

30 Mearsheimer, John J. "The false promise of international institutions." International Security 19.3 (1994): 5-49.
Bass, Gary J. "Atrocity \& Legalism.” Daedalus 132, no. 1 (2003): 73-82. http://www.jstor.org/stable/20027824. 2003.

Bull, H. La sociedad anárquica: un estudio sobre el orden en la política mundial. Madrid. Catarata. 1977.

Cassese, Antonio, Paola Gaeta, and John R. W. D Jones. The Rome Statute of the International Criminal Court : A Commentary. Oxford, England: Oxford University Press, 2002.

Cronqvist, Lasse. QCA Add-In. Version 1.1. Trier, 22.02.2019. https://www.qca-addin.net

De Nevers, Renee. "Imposing International Norms: Great Powers and Norm Enforcement." International Studies Review 9, no. 1 (2007): 53-80. http://www.jstor. org/stable/4621777.

Douglas, Lawrence. The Shrunken Head of Buchenwald: Icons of Atrocity at Nuremberg. Representations, (63), 39-64. doi:10.2307/2902917. 2018

Ellner, Steve. Journal of Latin American Studies 44, no. 1 (2012): 202-04. http://www.jstor.org.ezproxy.uniandes. edu.co:8080/stable/41349744.

Frahm, Ole. Report. Istituto Affari Internazionali (IAI), 2015. http://www.jstor.org/stable/resrep09759. 2015.

Goodliffe, Jay, Darren Hawkins, Christine Horne, and Daniel L. Nielson. "Dependence Networks and the International Criminal Court.” International Studies Quarterly 56, no. 1 (2012): 131-147. http://www.jstor.org. ezproxy.uniandes.edu.co:8080/stable/41409827.

Johns, Leslie. "The Diverging Theory and Practice of International Law." En Encyclopedia of Empirical International Relations Theory, 513-35. Oxford: Oxford University Press, 2018.

Keppler, Elise. "Managing Setbacks for the International Criminal Court in Africa." Journal of African Law 56, no. 1 (2012): 1-14. http://www.jstor.org/stable/41709948.

Krasner, Stephen D. Soberanía, hipocresía organizada. Paidós: Barcelona, 2001.

Lagon, Mark P. "The International System and the Reagan Doctrine: Can Realism Explain Aid to 'Freedom Fighters'?" British Journal of Political Science 22, no. 1 (1992): 39-70. http://www.jstor.org.ezproxy.uniandes. edu.co:8080/stable/193861. 
Mearsheimer, John J. "The false promise of international institutions." International Security 19.3 (1994): 5-49.

Meron, Theodor. "Judicial Independence and Impartiality in International Criminal Tribunals." The American Journal of International Law 99, no. 2 (2005): 359-69. doi:10.2307/1562502.

Nelson, Marcel. "Institutional Conflict and the Bolivarian Revolution: Venezuela's Negotiation of the Free Trade Area of the Americas." Latin American Perspectives 40, no. 3 (2013): 169-83. http://www.jstor.org. ezproxy.uniandes.edu.co:8080/stable/23466010.

Nossal, Kim Richard. "International Sanctions as International Punishment." International Organization 43, no. 2 (1989): 301-22. http://www.jstor.org.ezproxy.uniandes. edu.co:8080/stable/2706704.

Olatunde J. B. Ojo. "Nigeria and the Formation of ECOWAS." International Organization 34, no. 4 (1980): 571-604. http://www.jstor.org.ezproxy.uniandes.edu. co: $8080 /$ stable/2706513.

Pérez-Liñán, Aníbal. El método comparativo y el análisis de configuraciones causales. Latin American Review of Comparative Politics, 3, 2010.

Quesada Alcalá, Carmen. La Corte Penal Internacional Y La Soberanía Estatal. Tirant Monografías, 383. Valencia: Tirant Lo Blanch, 2005.

Ratner, Steven R. "International Law: The Trials of Global Norms." Foreign Policy, no. 110 (1998): 65-80. doi:10.2307/1149277.

Rudolph, Christopher. Power and Principle: The Politics of International Criminal Courts. Ithaca; London: Cornell University Press, 2017. http://www.jstor.org.ezproxy. uniandes.edu.co:8080/stable/10.7591/j.ctt1qv5q0z.

Sampford, Charles. "A Feuerbachian Inversion: From Sovereign Rights and Subjects Duties to Citizen Rights and State Duties." Security Challenges 7, no. 4 (2011): 51-60. https://www-jstor-org.ezproxy.uniandes.edu. co: $8443 /$ stable/26467116.

Schneider, Ben Ross. "Democratic Consolidations: Some Broad Comparisons and Sweeping Arguments." Latin American Research Review 30, no. 2 (1995): 215-34. http://www.jstor.org.ezproxy.uniandes.edu. co:8080/stable/2503840.

Shany, Yuval. Assessing the Effectiveness of International Courts: A Goal-Based Approach. American Journal of International Law 106, Nr. 2: 225-270. doi:10.5305/ amerjintelaw.106.2.0225, 2012.

Werle, Gerhard, Florian Jessberger, Claudia Cárdenas Aravena, Julia Geneuss, and María Gutiérrez Rodríguez. Tratado De Derecho Penal Internacional. Tercera Edición ed. Tirant Lo Blanch. Tratados. Valencia: Tirant Lo Blanch, 2017. 
Para publicar na Revista de Direito Internacional, acesse o endereço eletrônico www.rdi.uniceub.br ou www.brazilianjournal.org.

Observe as normas de publicação, para facilitar e agilizar o trabalho de edição. 